\title{
EXTRAPERITONEOSCOPIC RADICAL PROSTATECTOMY AFTER PELVIC SUGERY PROCEDURES
}

DOI: 10.36740/WLek202006102

\author{
Yuriy P. Serniak', Andriy I. Sagalevych², Oleksandr S. Frolov², Petr Y. Serniak', Mykola S. Kryvopustov ${ }^{3}$ \\ 1 UROLOGICAL CLINIC OF PROFESSOR SERNIAK, KYIV, UKRAINE \\ ${ }^{2}$ SHUPYK NATIONAL MEDICAL ACADEMY OF POSTGRADUATE EDUCATION, KYIV, UKRAINE \\ ${ }^{3}$ 0. 0. BOGOMOLETS NATIONAL MEDICAL UNIVERSITY, KYIV, UKRAINE
}

\begin{abstract}
The aim of study is to evaluate the results of extraperitoneoscopic radical prostatectomy performed in the presence of various complicating factors. Materials and methods: This prospective study included 7 patients with a diagnosis of prostate cancer (T1-T2b, №, Mo) who underwent extraperitoneoscopic radical prostatectomy (ERPE).

Results: Among all ERPEs performed on seven patients, the maximum duration of the surgery was 6 hours 30 minutes, and the minimum one was 3 hours 40 minutes. The average volume of blood loss did not exceed $350 \pm 20 \mathrm{ml}$; decline in hemoglobin level was in the range of 10-12 $\mathrm{g} / \mathrm{l}$. The urethral catheter was removed no earlier than $7 \mathrm{days}$ after the surgery. Drainage from the space of Retzius was removed on the $3^{\text {rd }}$ day. Postoperative complications occurred in 2 patients (28.57\%). The maximum period of postoperative outpatient follow-up was 22 months. In 6 patients (85.71\%), complete urinary retention up to 3 months was noted. One patient (14.29\%) had mild urinary incontinence (PADtest - no more than one pad per day). In these cases, sexual potency was absent in $100 \%$ of patients. The maximum postoperative PSA level in the group reached $0.13 \mathrm{ng} / \mathrm{ml}$. Conclusions: 0 wn experience of ERPE in patients on the background of previous transurethral resections and vaporization of the prostate demonstrated the effectiveness and safety of the method. More extensive studies with a larger number of cases are needed.
\end{abstract}

KEY WORDS: localized prostate cancer, radical prostatectomy, urinary retention, transurethral resection

Wiad Lek. 2020;73(6):1093-1096

\section{INTRODUCTION}

Benign prostatic hyperplasia (BPH) is one of the most common diseases in elderly men. Despite the preoperative screening of patients to exclude prostate cancer, the latter is often a histological finding after TURP for BPH [1].

Radical prostatectomy (RPE) in clinically localized prostate cancer is a safe procedure and successfully reduces the risk of death from cancer [2]. Many factors, including the previous surgery on organs of the lower part of the abdominal cavity, pelvis or prostate gland, as well as radiation therapy, change the anatomy of the para-prostatic space, which, together with the experience of the surgeon, determine the possibility of successful RPE. In particular, the previous surgery or radiation therapy in this area can lead to the formation of connective tissue fibrosis that drastically changes the anatomy of interfascial spaces and the ratio of tissue layers, which complicates the performance of RPE to a great extent [3].

Some researchers state that the previous extensive transabdominal or pelvic surgery is a contraindication for laparoscopic RPE [4]. However, other studies have shown that RPE can be safely performed after prostate surgery [5], even if extensive fibrosis is observed and interfascial dissection planes are absent. Many studies have shown that the retropubic RPE and laparoscopic RPE are difficult to perform after TURP, because perforation of the prostatic capsule during TURP with extravasation of blood and irrigation fluid can result in periprostatic fibrosis, sclerotic changes in the capsule of the prostate and distortion of the surgical planes [6].

Thus, in the modern literature there is a lot of conflicting information about the results of laparoscopic and open RPE in patients who have previously undergone pelvic surgery. This fact encouraged us to describe our experience in performing extraperitoneoscopic radical prostatectomy (ERPE) after various surgical procedures on the lower abdominal and pelvic organs.

\section{THE AIM}

The aim of the study is to evaluate the results of extraperitoneoscopic radical prostatectomy performed in the presence of various complicating factors.

\section{MATERIALS AND METHODS}

Our prospective study included 7 patients with a diagnosis of prostate cancer (T1-T2b, No, Mo), who underwent extraperitoneoscopic radical prostatectomy (ERPE). The patients underwent surgeries from 2016 to 2018 , by the same surgeon 
Table 1. Patients' distribution

\begin{tabular}{cc}
\hline Laser vaporization of the prostate & 1 (patient№1) \\
\hline Laparotomy for peritonitis, appendectomy, right-sided hernioplasty. & 1 (patient№2) \\
\hline Epicystostomy & 1 (patient№3) \\
\hline Transvesical open prostatectomy & 1 (patient№4) \\
\hline Bipolar TURP & 3 (group of patients№5) \\
\hline
\end{tabular}

(experience in ERPE about 500 surgeries). The inclusion criteria for patients are the following: medical history of surgeries on the organs of the abdominal cavity and small pelvis, endoscopic surgery on the prostate gland. Patients were distributed as follows: 1 patient after laser vaporization of the prostate (patient No. 1); 1 patient after laparotomy for peritonitis, appendectomy, right-sided hernioplasty (patient No. 2); 1 patient with an epicystostomy inserted for bladder obstruction as a result of prostatic hyperplasia (patient No. 3); 1 patient after transvesical open prostatectomy (patient No. 4); 3 patients after bipolar TURP (group of patients No. 5). Table I. It is worth noting that all these patients underwent the first surgeries in other medical institutions. In patient No. 1, the preoperative PSA level was $8.6 \mathrm{ng} / \mathrm{ml}$, in patient No. 2 - 5.5 $\mathrm{ng} / \mathrm{ml}$, in patient No. $3-6.2 \mathrm{ng} / \mathrm{ml}$, in patient No. $4-5.4 \mathrm{ng} /$ $\mathrm{ml}$. In patients who previously underwent TURP: in the first patient, the preoperative level of total PSA was $3.2 \mathrm{ng} / \mathrm{ml}$, in the second patient it was $1.23 \mathrm{ng} / \mathrm{ml}$, and in the third patient it was $2.2 \mathrm{ng} / \mathrm{ml}$. In all 7 patients histologic examination revealed a tumor of the prostate. The sum of points on the Gleason score in patient No. 1 was $3+4=7$, in patient No. 2 it was $4+4=8$, in patient No. 3 the score was $3+4=7$, in patient No. 4 it showed $4+3=7$. In group No. 5 : one patient had $3+4=7$, the second $-3+3=6$ and the third $-4+3=7$. Before ERPE, in patient No. 1 the prostate volume was $63 \mathrm{~cm}^{3}$, in patient No. $2-52 \mathrm{~cm}^{3}$, in patient No. $3-86 \mathrm{~cm}^{3}$, in patient No. $4-27 \mathrm{~cm}^{3}$. In patients' group No. 5, one patient had the prostate volume of $12 \mathrm{~cm}^{3}$, the second $-16 \mathrm{~cm}^{3}$, and the third $-11 \mathrm{~cm}^{3}$. The minimum time period after the first surgery before ERPE was 2 weeks, the maximum one was 5 years. All patients of group No. 5 were diagnosed with a prostate tumor immediately after transurethral resection during histological examination of postoperative material. In all other cases, it happened after transrectal puncture biopsy for an elevated PSA level. In all the observations included in this study, we used the Rocco stitch as a stage of posterior reconstruction, as well as the stitching of the bladder to puboprostatic ligaments as a stage of anterior reconstruction.

The preoperative examination of patients before performing the ERPE was carried out in accordance with modern standards and recommendations of the European Society of Urology and included a thorough medical history, physical, laboratory and instrumental types of examination. To exclude common forms of prostate cancer, magnetic resonance imaging of the small pelvis was performed, which confirmed the stage of the disease; the absence of damage to regional lymph nodes and the absence of distant metastasis. In our opinion, more extensive observations are required, followed by statistical studies.

\section{RESULTS AND DISCUSSION}

Among all ERPEs performed on seven patients, the maximum duration of the surgery was 6 hours 30 minutes, and the minimum one was 3 hours 40 minutes. There were no conversions from extraperitoneoscopic access to "open" one. We did not perform pelvic lymphadenectomy in those cases due to the absence of indications. All surgeries were conducted in accordance with oncological requirements; histological examination of the preparations did not show "positive" surgical margin. In all cases, taking into consideration the manifested postoperative fibrosis, extrafascial prostatectomy was performed. Intraoperative complications (injuries of the rectum, the ureters, and great vessels), fragmentation of the prostate or damage to its capsule were avoided. The average volume of blood loss did not exceed $350-20 \mathrm{ml}$; decline in hemoglobin level was in the range of $10-12 \mathrm{~g} / \mathrm{l}$. There was no need for blood transfusion. All patients were activated on the first postoperative day. The urethral catheter was removed no earlier than 7 days after the surgery. Drainage from the space of Retzius was removed on the $3^{\text {rd }}$ day. Postoperative complications occurred in 2 patients (28.57\%). Due to the lack of tightness of the anastomosis in 2 patients (patient No. 1 and No. 4), the urethral catheter was removed on the $14^{\text {th }}$ day after the control cystography. All patients were discharged for outpatient treatment with recovery. The maximum period of postoperative outpatient follow-up was 22 months. In 6 patients (85.71\%), complete urinary retention up to 3 months was noted. One patient (14.29\%) had mild urinary incontinence (PAD-test - no more than one pad per day). In these cases, sexual potency was absent in $100 \%$ of patients. The maximum postoperative PSA level in the group reached $0.13 \mathrm{ng} / \mathrm{ml}$.

It is common knowledge, in most patients who have previously undergone open surgery, tissue fibrosis develops in the area of the surgery, which, when performing subsequent surgeries, causes a number of "technical" difficulties for the surgeon and this contributes to intraoperative complications [9]. We also encountered such "technical" difficulties in performing ERPEs against the background of previous surgical procedures, where infiltrative and cicatricial interfascial fibrotic changes took place to one degree or another.

Thus, after an open adenomectomy in the patient (taking into consideration that the surgery was performed via transvesical access and the bladder wall was "soldered" with paravesical fatty tissue), we needed to access the Retzius space with excision of postoperative scars (Figure 1).

After that, the Retzius space was marked and under direct vision (according to the Hasson technique), trocars were placed and the wound was sutured layer by layer. The further 


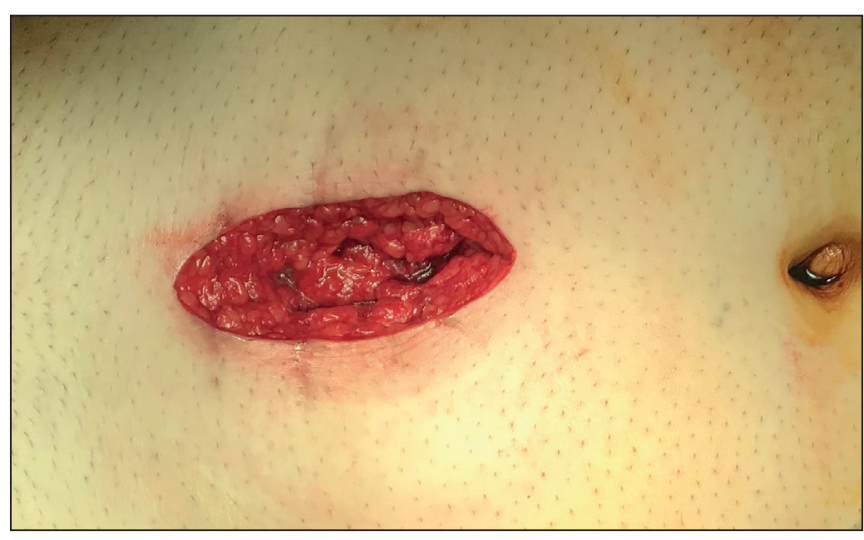

Fig. 1. The formation of extraperitoneoscopic access. The postoperative scar in the patient after transvesical adenomectomy was excised.
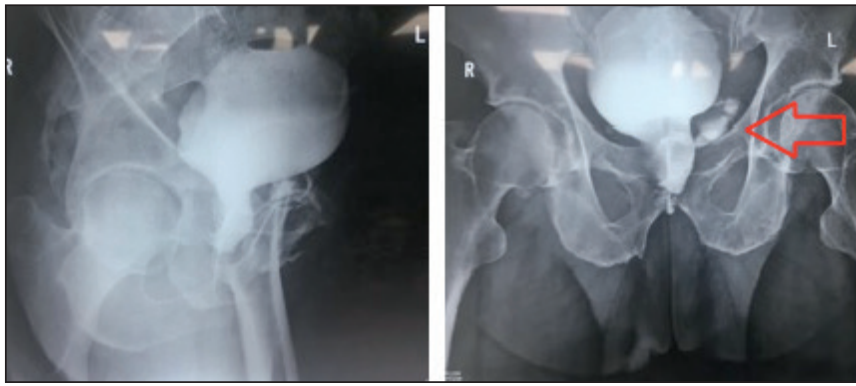

Fig. 3. Cystogram of the patient G.: direct and lateral projection.

surgery progress did not differ from the standard ERPE. In both cases, we had to perform the conversion from extraperitoneoscopic access to laparoscopic one. In the first case, ERPE was performed in patient No. 2, in the second observation it was in patient No. 4. It is worth noting that in these cases we did not use cylindrical dissectors at the stage of marking of the Retzius space to place an optic trocar, but performed this step with the index finger from a small incision under the umbilical ring up to $2 \mathrm{~cm}$. In our opinion, this allows us to perform this stage more precisely and minimizes the chance of trauma to the peritoneum. Nevertheless, the need for conversion was determined by a trauma of the peritoneum at the stage of finger marking of the Retzius space. This in turn led to the ingress of carbon dioxide into the abdominal cavity and, as a result, squeezing our workspace. After the conversion from extraperitoneoscopic access to laparoscopic one, we noted that the greater omentum was "soldered" to the anterior abdominal wall, and the ileocecal angle of the large intestine was medially displaced and adjacent to the rectum. This made us separate the above-indicated structures from each other, for safe access to the Retzius space. After opening the parietal peritoneum, the progress of the surgery did not differ from the standard laparoscopic radical prostatectomy. From our point of view, an important factor determining the success of these surgical procedures is the experience of a surgeon who is aware of all access options for performing ERPE.

In other 4 cases, in patients with a medical history of endoscopic surgery (patient No. 1 and patient group No. 5), infiltrative and cicatricial periprostatic changes were most observed and combined with fibrosclerotic changes in interfascial

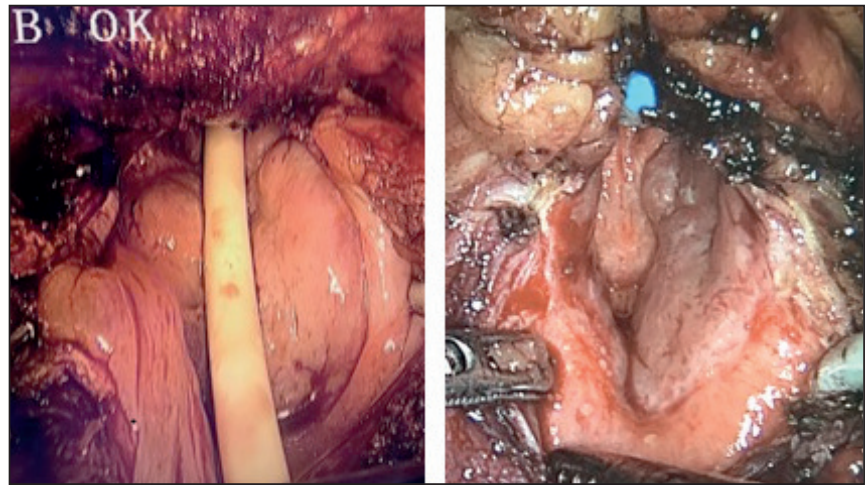

Fig. 2. The prostatic urethra region after dissection of the bladder.

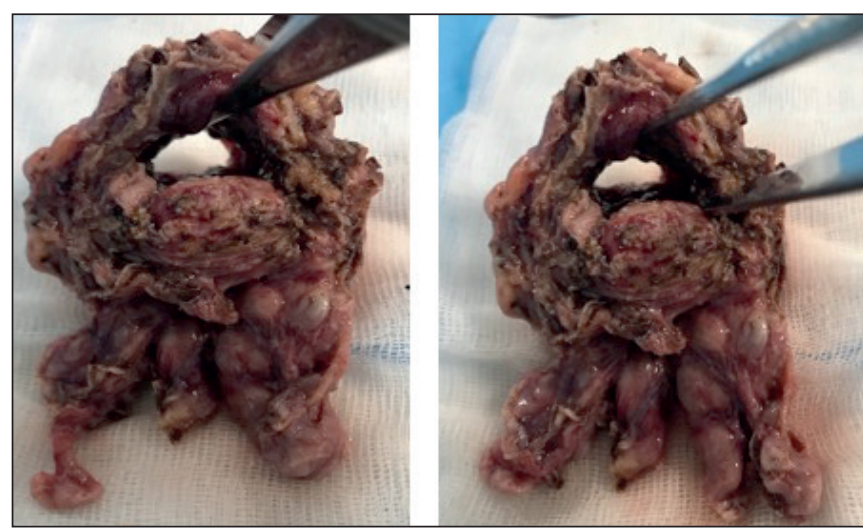

Fig. 4. Gross specimen of the patient $\mathrm{G}$. The prostate with seminal vesicles and sections of the vas deferens as a single unit.

anatomy of the small pelvis, which significantly complicated extrafascial prostate excretion. Therefore, in our observations, we noticed a severe cicatricial deformity of the bladder neck, fixed to the pubic articulation along the anterior semicircle; significant periprostatic infiltrative-cicatricial changes; asymmetry of the sclerosed prostate and the lack of the possibility of interfascial dissection due to cicatricial changes in the anatomical layers. The density of scar tissue did not allow to clearly differentiate the border between the prostate and the bladder neck (Figure 2). The latter required a wide excision with subsequent reconstruction of the bladder neck. In all cases, navigation was carried out along the cylinder of the urethral catheter during its traction.

In our opinion, this stage of the operation is associated with a high risk of damage to the orifices of the ureters due to the wide excision of the bladder neck. Taking into consideration that fact, we performed that stage precisely and the search of the orifices in the deformed vesical triangle took a long time (up to 30 minutes). Clear visualization of the orifices is important in the formation of the posterior semicircle of the vesicourethral anastomosis. Therefore, in our opinion, perhaps, as prevention of such complications in patients of this category, preoperative ureteral stenting should be performed.

In 3 cases ( 2 patients of group No. 5 and patient No. 1), we were not able to differentiate the anterior surface of the prostate and the dorsal venous complex in scar tissue. The latter was not stitched; the tissues were cut with "cold" scissors, sliding along the surface of the pubic articulation to minimize injury 
to the external urethral sphincter. In this case, no bleeding was observed or it was minimal, not requiring through-out suturing of the dorsal venous complex. The described changes, apparently, are determined by the technological features of previously conducted TURP and vaporization. Thus, it is known that transurethral resection of adenomatous nodes is often accompanied by perforation of the surgical capsule of the prostate and the coagulation of bleeding vessels and vaporization of tissues only aggravate the inflammatory changes.

\section{EXAMPLE: PATIENT NO 1.}

Patient G., 63 years old, complained of pain in the suprapubic region on the left. Medical history: 3 months ago laser vaporization of the prostate. PSA before surgery was $3.9 \mathrm{ng} /$ $\mathrm{ml}$; prostate biopsy was not performed. Examination: there is no ultrasound of ectasia of the calices-pelvis system (CPS) on both sides, there is no residual urine. After emptying the bladder there is a liquid formation of $5 \times 4 \mathrm{~cm}$ in a small pelvis on the left. Cystography is performed (Figure 3) in the direct and lateral projections: in the direct projection, the bladder is uniformly contrasted, there are no filling defects. In the area of the prostatic urethra on the left, the flow of contrast medium into the Retzius space of $6 \times 4 \mathrm{~cm}$ is noticed.

3 months after vaporization PSA was $8.6 \mathrm{ng} / \mathrm{ml}$. A transrectal biopsy of the prostate was performed: prostatic adenocarcinoma $4+4=8$ points on the Gleason score. The volume of the prostate was $63 \mathrm{~cm}^{3}$. Endoscopic extraperitoneal extrafascial radical prostatectomy was performed. Surgery duration was 4 hours 50 minutes, blood loss $-320 \mathrm{ml}$. The drainage was removed on the $3^{\text {rd }}$ day; the urethral catheter was removed on the $9^{\text {th }}$ day. During histological examination, the surgical margin was negative (Figure 4). Urinary stress incontinence of mild severity (up to 1 pad per day) was noted for up to 3 months. After 3 months, urine is completely retained.

Having performed these surgical procedures, we noted that the severity of the adhesive process in the early stages (up to one month) was less than at a later date. In our observations, we managed to avoid serious intraoperative complications such as the rectum trauma, the ureters trauma, massive blood loss. However, periprostatic adhesion due to the previous surgery complicates the excretion of seminal vesicles and the identification of neurovascular bundles in the projection of the crura of the prostate, which forces an extrafascial RPE to be performed without preserving the neurovascular bundles, thereby increasing the risk of impotence and urinary incontinence.

\section{CONCLUSIONS}

Previous pelvic surgery should not be considered an absolute contraindication for performing ERPE. The determining factor for success in performing these surgical procedures is the experience of the surgeon. Own experience of ERPE in patients against the background of previous transurethral resections and vaporization of the prostate showed the effectiveness and safety of the method. In patients after "open" surgeries on the prostate, it is advisable to refuse extraperitoneoscopic access. Having performed these surgical procedures, we noted that the severity of the adhesive process in the early stages (up to one month) was less than at a later date. In our opinion, it is recommended for patients after endoscopic surgeries on the prostate to begin the surgery with cystoscopy and stenting in cases of close locations of the orifices to the bladder neck. However, taking into account the small amount of data from clinical cases, more extensive research is needed in this direction.

\section{REFERENCES}

1. Shamloul R., Ghanem H. Erectile dysfunction. The Lancet. 2013;381 (9861):153-165. doi:10.1016/50140-6736(12)60520-0

2. Bill-Axelson A., Holmberg L., Ruutu M. et al. Radical prostatectomy versus watchful waiting in early prostate cancer. N Engl J Med 2005;352:1977-84.

3. Colombo R., Naspro R., Salonia A. et al. Radical prostatectomy after previous prostate surgery: clinical and functional outcomes. J Urol 2006;176(6 Pt 1):2459- 63.

4. Rassweiler J., Sentker L., Seemann 0. et al. Laparoscopic radical prostatectomy with the Heilbronn technique: an analysis of the first 180 cases. J Urol 2001;166:2101-8.

5. Palisaar J.R., Wenske S., Sommerer F. et al. Open radical retropubic prostatectomy gives favourable surgical and functional outcomes after transurethral resection of the prostate. BJU Int 2009;104:611-5.

6. Teber D., Cresswell J., Ates M. et al.: Laparoscopic radical prostatectomy in clinical T1a and T1b prostate cancer: oncologic and functional outcomes-a matched-pair analysis. Urology 2009; 73: 577-581. Epub 2008 Dec 18.

The work is a fragment of the planned research work of the Department of Urology of the Shupyk National Medical Academy of Postgraduate Education "Improvement of surgical technique in performing laparoscopic radical prostatectomy in patients with localized prostate cancer".

\section{ORCID and contributorship:}

Yuriy P. Serniak - 000-0002-2146-4715 ${ }^{A}$

Oleksandr S. Frolov - 0000-0002-4608-8613 ${ }^{B}$

Petr Y. Serniak - 0000-0003-2989-8929B

Mykola S. Kryvopustov - 0000-0003-4978-4873 ${ }^{C}$

Oleksandr S. Frolov - 0000-0002-4608-8613 ${ }^{D}$

Andriy I. Sagalevych - 0000-0001-5273-6907 ${ }^{E}$

Yuriy P. Serniak - 0000-0002-2146-4715 ${ }^{F}$

\section{Conflict of interest:}

The Authors declare no conflict of interest.

\section{CORRESPONDING AUTHOR \\ Oleksandr S. Frolov}

Department of Urology

Shupyk National Medical Academy of Postgraduate Education

Dorohozhytska Street, 9, 04112, Kyiv, Ukraine

tel: +380953516624

e-mail:frolov_alexandr@ukr.net

Received: 25.01 .2020

Accepted: 08.05 .2020 